\title{
Carotid Artery Segmentation Using an Outlier Immune 3D Active Shape Models Framework
}

\author{
Karim Lekadir and Guang-Zhong Yang \\ Visual Information Processing Group, Department of Computing \\ Imperial College London, United Kingdom \\ \{lekadir, gzy\}adoc.ic.ac.uk
}

\begin{abstract}
This paper presents an outlier immune 3D active shape models framework for robust volumetric segmentation of the carotid artery required for accurate plaque burden assessment. In the proposed technique, outlier handling is based on a shape metric that is invariant to scaling, rotation and translation by using the ratio of inter-landmark distances as a local shape dissimilarity measure. Tolerance intervals for each descriptor are calculated from the training samples and used to infer the validity of landmarks. The identified outliers are corrected prior to the model fitting using the ratios distributions and appearance information. To improve the feature point search, the method exploits the geometrical knowledge from the outlier analysis at the previous iteration to weight the gray level appearance based fitness measure. A combined intensity-phase feature point search is also introduced which significantly limits the presence of outliers and improves the overall search accuracy. Both numerical and in vivo assessments of the method involving volumetric segmentation of the carotid artery have shown that the outlier handling technique is capable of handling a significant presence of outliers independently of the amplitudes.
\end{abstract}

\section{Introduction}

Atherosclerosis is central to the clinical sequelae of coronary heart, cerebrovascular and peripheral vascular disease. For the assessment of atherosclerosis, cardiovascular MR is emerging as an attractive tool clinically because of its ability in identifying arterial remodeling, as well as providing information on plaque composition in vivo. The technique, however, is susceptible to motion and blood flow artifact and consistent plaque burden assessment involving the calculation of total vessel volume in response to therapeutic measures is a significant challenge. This is because small, progressive changes of the vessel volume over time require a high segmentation accuracy, and the traditional manual approach is subject to significant inter- and intraoperator variabilities. Most existing segmentation methods for the carotid artery segmentation [1-3] are semi-automatic and usually based on Active Contours. They require additional user interaction to correct for errors due to artifacts and the lack of a model to limit the segmentation results to valid instances.

Recently, automatic segmentation based on statistical shape modeling has attracted significant interests for vessel segmentation because of its unique strengths in exploit- 
ing a priori knowledge about the geometry of known anatomical shapes. The Active Shape Models (ASMs) [4], for example, can capture spatio-temporal principal shape variations from a set of labeled examples based on a Point Distribution Model (PDM). The model is then fitted to unseen shapes in an iterative manner where the pose and shape parameters are estimated using a least squares minimization approach. The ASM, however, is known to be problematic in the presence of image artifacts as well as incomplete or complex image features. In this case, some of the landmarks generated at the feature point detection stage are erroneous as they lie on incorrect boundary positions. These outlying landmarks are known to affect considerably the segmentation accuracy using the ASM search. A number of modifications have been introduced [5-8] to limit the effects of the outliers on the ASM search, but their actual performance is compromised as the number and amplitude of the outliers increase, especially for volumetric segmentation.

The purpose of this paper is to present a robust outlier handling method for volumetric segmentation with ASM [9]. The method detects and corrects the individual outliers prior to the estimation of the pose parameters by using an invariant shape metric based on the ratio of inter-landmark distances. Tolerance intervals are calculated at the training stage for each descriptor to detect invalid ratios, and thus the corresponding erroneous landmarks. Instead of rejecting the identified outliers or replacing them by the corresponding mean values, they are rectified by a combined use of the ratios distributions and appearance information. The geometrical knowledge gathered from the outlier analysis is propagated to successive iterations for feature point detection. This increases the search accuracy and reduces the number of iterations needed for final convergence. We also present a combined intensity-phase based feature point search, which significantly limits the presence of outliers and improves the overall accuracy. The practical value of the technique is validated by using a newly developed combined intensity-phase data acquisition scheme of plaque burden assessment for carotid arteries.

\section{Methods}

\subsection{Outlier Handling}

The ASM search involves in an iterative manner the detection of feature points [10] which are then fitted to the model by the estimation of pose and shape parameters. This is achieved using least squares minimizations which are known to be sensitive to extreme values. Thus, when outliers are present amongst the feature points, the ASM often introduce significant errors in the segmentation results. Without the correct estimation of the pose parameters, it is difficult to infer the presence of outliers due to residuals distribution introduced. Thus, the proposed outlier detection is based on a fully invariant shape descriptor using the ratio of interlandmark distances, which allows the decoupling of outlier analysis and ASM model fitting. This metric can be calculated for any triplet of points and represents the relative position of a landmark with regard to other points in the shape. An important property of this descriptor is its ability to perform as a geometrical measure of dissimilarity, which makes it ideal for 
outlier detection. Each ratio $r_{i j k}$ can be assumed to follow a Gaussian distribution and we use $\bar{r}_{i j k}$ and $s_{i j k}$ to represent the mean and standard deviation of the distribution. The idea behind the proposed method is that outliers have some of their associated ratios as extreme values. Thus, outlier detection is achieved by using tolerance analysis [11], with which tolerance intervals are calculated for each ratio from the training samples by using the following equation:

$$
T_{i j k}=\left[\bar{r}_{i j k}-k_{2} \cdot s_{i j k}, \bar{r}_{i j k}+k_{2} \cdot s_{i j k}\right]
$$

where $k_{2}$ is the two-sided tolerance factor [9] representing the size of the interval. From each tolerance interval, a likelihood ratio measure $f_{r}$ can be derived, i.e.,

$$
f_{r}\left(r_{i j k}\right)=\left\{\begin{array}{cc}
1 & \text { if } r_{i j k} \in T_{i j k} \\
0 & \text { elsewhere }
\end{array}\right.
$$

This measure is equal to 0 if the ratio is an extreme value but it does not indicate which of the three landmarks associated with the ratio is invalid. By summing all the ratio likelihood measures that a given landmark is associated with, however, the likelihood of the point being an inlier can be estimated:

$$
f_{p}\left(P_{i}\right)=\frac{1}{K_{i}} \sum_{j, k} f_{r}\left(r_{i j k}\right) f\left(r_{j k i}\right) f\left(r_{k i j}\right)
$$

where $K_{i}$ is the number of ratios associated with the landmark $P_{i}$.

Based on the above likelihood measure, an iterative algorithm is used where one outlier is identified at each iteration. When no outliers are present, all the likelihood measures of the feature points will be close to 1 . In the presence of outliers, however, the likelihood measures decrease from 1, and the amount of decrease is more significant for the outliers. Thus, at each iteration, the landmark with the lowest likelihood measure is rejected and the likelihood measures of the remaining landmarks are updated by subtracting the contribution of the rejected point. This procedure is repeated until the lowest likelihood measure is close to 1 , which suggests that all the remaining landmarks are inliers.

The derived inliers are then used to rectify the detected outliers by using a local search regime that maximizes the product of the p.d.f. of the associated ratios. This is equivalent to choosing the point that minimizes the following least-square function:

$$
\sum_{j, k}\left(\frac{r_{i j k}-\bar{r}_{i j k}}{s_{i j k}}\right)^{2}=\sum_{j, k}\left(\frac{d_{i j}-d_{j k} \bar{r}_{i j k}}{d_{j k} s_{i j k}}\right)^{2}
$$

where $d$ corresponds to interlandmark distances. It should be noted that the correction suggested by Eq. (5) is only based on geometrical information and may not optimally correspond to local salient features. Therefore, a final local adjustment based on the gray level information is required. Subsequently, the residual distribution can be seen as Gaussian and the traditional ASM model fitting can be applied efficiently. 


\subsection{Improved Feature Point Detection}

In this paper, two strategies are introduced to improve the feature point search. The first approach is directly connected to the outlier handling method described above and uses the geometrical knowledge gathered from the outlier analysis at previous iteration to weight the intensity fitness measure $d_{g}$ as follows:

$$
f_{\text {total }}\left(P_{j}\right)=d_{g}\left(P_{j}\right) \cdot w\left(P_{j}\right)
$$

where the weights $w$ take values from 0 to 1 and are calculated by using the point likelihood measure in Eq. (4) for each candidate point. This enables regions around the true position of the landmark point to be heavily weighted to avoid potential outliers from being generated. More importantly, this approach makes the segmentation procedure more stable as only a small number of iterations are needed for final convergence.

To further improve the system accuracy, feature point search based on combined intensity-phase information is also introduced. In cardiovascular MR, the assessment of plaque burden is usually achieved with 3D Turbo Spin-Echo (TSE) sequences. To suppress blood flow artifacts, double inversion preparation on either side of the excitation slab is introduced. Three-dimensional TSE techniques have the advantage of providing contiguous coverage and high SNR, but they can suffer from incomplete blood signal suppression in certain regions. However, it has been shown recently that by the introduction of velocity phase sensitivity to the sequence, it is possible to use the reconstructed phase images to separate blood signal from vessel wall boundaries [12]. This additional information can also be used for a more robust feature point search to further limit the presence of outliers. This is achieved by combining the gray level appearance with the phase information to construct a fitness measure for feature point search based on the phase profile $\varphi$ :

$$
\varphi=\left(\left|\varphi_{1}-\varphi_{s}\right|, \ldots,\left|\varphi_{h}-\varphi_{s}\right|\right)
$$

where $h$ is the size of the profiles and $\varphi_{s}$ the stationary phase (usually equal to 0 ). Both the intensity and phase profiles are then normalized as follows:

$$
\hat{g}_{i}=\frac{g_{i}-g_{\min }}{g_{\max }-g_{\min }} \quad \hat{\varphi}_{i}=\frac{\varphi_{i}-\varphi_{\min }}{\varphi_{\max }-\varphi_{\min }}
$$

After normalization, the intensity and phase vectors are concatenated to form a combined profile vector $p$. PCA is then applied to the combined profile vectors of the training set for each individual landmark to extract the mean profile and the principal modes of variation. The Mahalanobis distance is then used as in the original ASM formulation [10] to calculate the global fitness measure as follows:

$$
(p-\bar{p}) S_{p}^{-1}(p-\bar{p})
$$

Other strategies can also be used to combine appearance and phase information, such as the use of a $k \mathrm{NN}$ classification approach as proposed in [13]. 


\subsection{Experiments}

To assess the practical value of the algorithm, 25 carotid artery datasets were collected. Subjects with carotid artery atherosclerosis, as shown by prior carotid Doppler ultrasonography, underwent two CMR scans. Both asymptomatic and symptomatic subjects were included. MR was performed using a 1.5T scanner (Sonata, Siemens, Erlangen Germany), a purpose-built two-element phased-array surface carotid coil and a specially designed head and neck cushion for immobilization. A 3D volumeselective TSE sequence was used with a pixel size of $0.47 \mathrm{~mm}^{2}$, slice thickness of 2 $\mathrm{mm}$. A velocity phase sensitivity of $\pi / 40 \mathrm{rad} / \mathrm{cm} / \mathrm{s}$ was used to acquire the phase images. For each dataset, 20 slices around the bifurcation were selected as a region for measurement. Delineations by an expert were performed using 272 landmarks. The data sets used for training and evaluation were selected on a leave-one-out basis. To tackle the problem of over-fitting and also to take into account the topological and geometrical configuration of the carotid artery including the main, internal and external branches, an ASM formulation similar to [14] was used such that points were grouped into segments at different cross sections of the branches.

\section{Results}

For a detailed assessment of the performance of the outlier handling algorithm in the presence of different levels of outliers, the following experiments were carried out by randomly adding synthetic outliers to the manual delineations using non-Gaussian noise. In a first experiment, the percentage of the outliers varied from 0 to $65 \%$ of the total number of landmarks while the amplitude was fixed to $50 \%$ of the average extent of the arteries. The average and standard deviation point to surface error in Fig. 1(a) demonstrate that the proposed method can handle up to $50 \%$ of outliers. In a second experiment, the percentage of outliers was fixed to $25 \%$ while the maximum amplitude varied from 0 to $9.25 \mathrm{~mm}$. It can be seen from Fig. 1(b) that the performance of the method is independent of the extremity of the outliers.
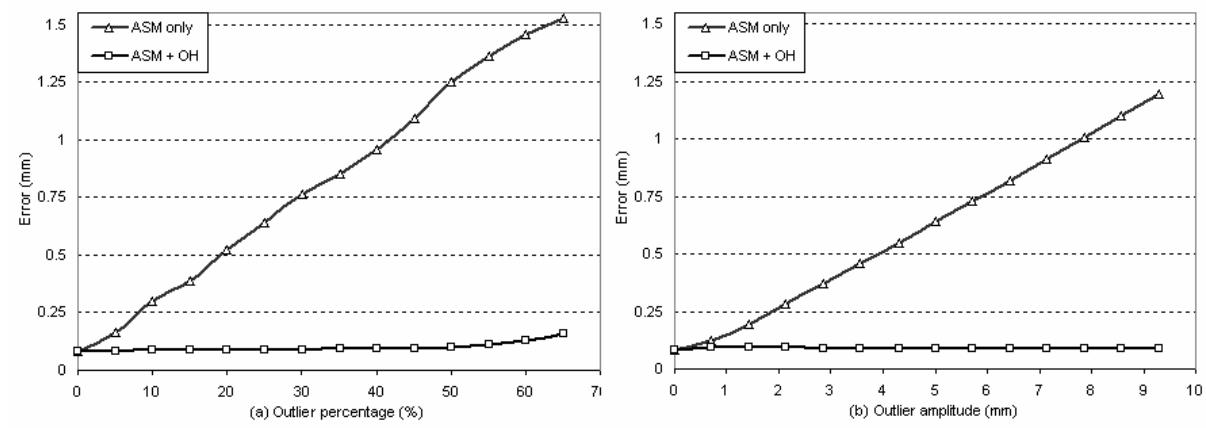

Fig. 1. Mean segmentation error of the traditional ASM and ASM with the proposed outlier handling $(\mathrm{ASM}+\mathrm{OH})$ in response to different percentage (a) and amplitude (b) of the outliers 


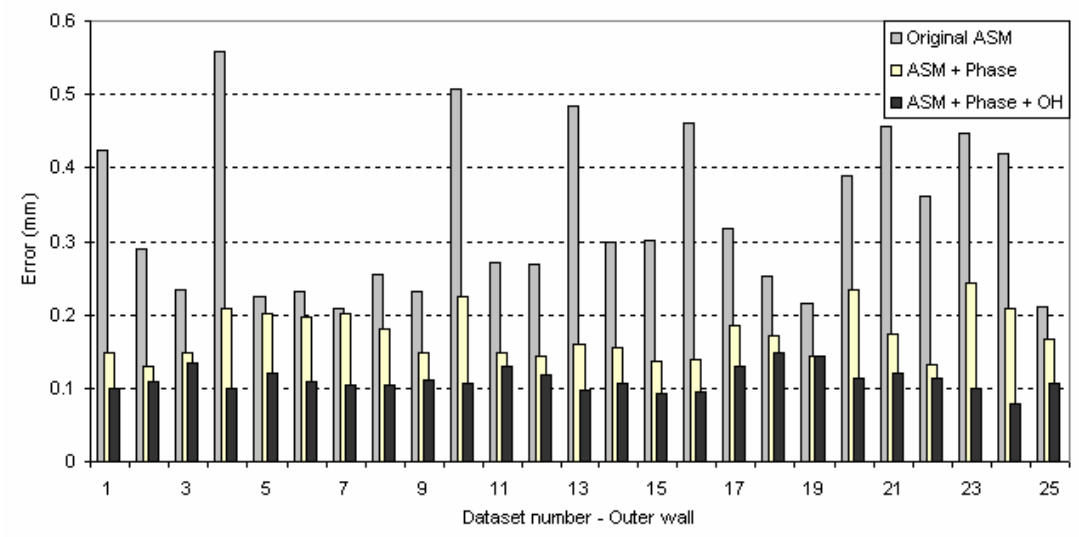

Fig. 2. Segmentation errors for the outer wall boundary by using the original ASM, ASM with the combined intensity-phase fitness measure (ASM+Phase), and the latter method with outlier handling (ASM+Phase+OH) for the 25 datasets used in this study
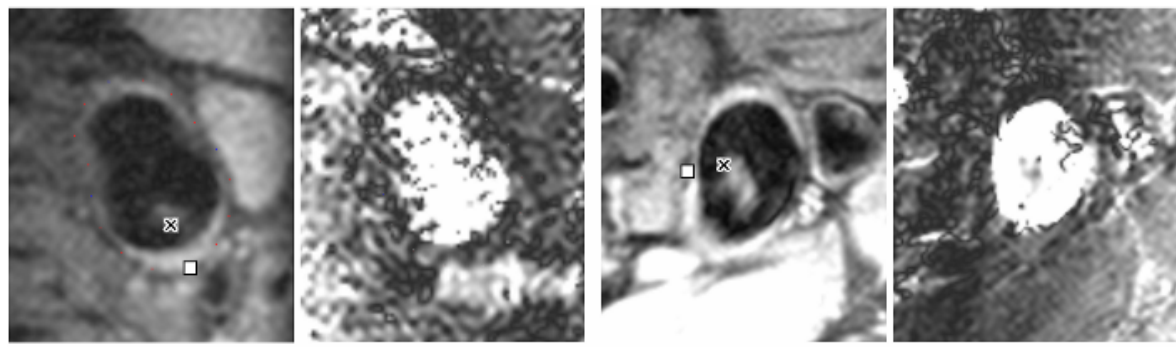

Fig. 3. 2D illustrations of the occurrence of the outliers (in crosses) due to blood flow artifacts present in the magnitude images and how the phase images at the right can be used for true boundary localization (in squares)

For in vivo validation, the original ASM and the proposed framework were applied to the 25 carotid artery datasets. Fig. 2 shows the segmentation results for the outer wall boundary which is particularly prone to outliers. Firstly, it can be seen that the combined intensity-phase feature point detection is considerably more robust as the average error decreases from $0.33 \pm 0.12 \mathrm{~mm}$ for the original ASM to $0.17 \pm 0.03 \mathrm{~mm}$ when using the proposed feature point search (47.91\% improvement). However, some outliers are still present amongst the feature points and further improvement is achieved using the proposed outlier handling scheme to a final average of $0.11 \pm 0.02$ $\mathrm{mm}$. This corresponds to $63.28 \%$ overall improvement of the original technique, which is significant for serial plaque burden assessment. Fig. 3 shows two examples taken at the bifurcation region, where outliers were generated by using the traditional intensity based fitness measure due to blood flow artifacts. It can be seen that the combined use of intensity and phase information is able to localize the correct boundary position. For 3D detailed visual illustration, Fig. 4 shows two examples of the application of the outlier handling procedure, followed by a robust generation of a 
plausible instance of the shape by the ASM. The accuracy of the proposed method for the luminal and vessel outer wall boundary as well as the volumetric results are summarized in Table 1, demonstrating the achievable accuracy in a clinical setting.
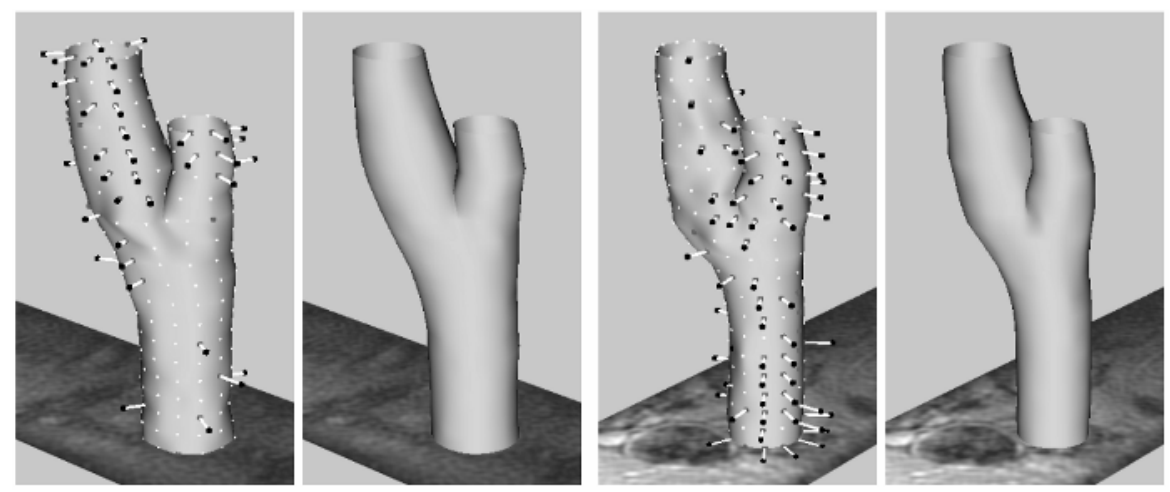

Fig. 4. Two 3D examples of the outlier handling algorithm, where the outliers (in black) are detected and corrected before the ASM model fitting procedure

Table 1. Overall segmentation accuracy of the patient data for the proposed technique

\begin{tabular}{lcccc}
\hline & $\begin{array}{c}\text { Average } \\
\text { Error }\end{array}$ & $\begin{array}{c}\text { Standard } \\
\text { deviation }\end{array}$ & $\begin{array}{c}\text { Median } \\
\text { Error }\end{array}$ & $\begin{array}{c}\text { Maximum } \\
\text { error }\end{array}$ \\
\hline Luminal boundary $(\mathrm{mm})$ & 0.09 & 0.01 & 0.09 & 0.13 \\
Outer wall boundary $(\mathrm{mm})$ & 0.11 & 0.01 & 0.10 & 0.14 \\
Vessel wall volume $(\%)$ & 3.19 & 2.43 & 2.13 & 8.64 \\
\hline
\end{tabular}

\section{Conclusions}

In this paper, we have presented an outlier immune framework for volumetric segmentation that is suitable for plaque burden assessment. The method is based on outlier detection and correction prior to the model fitting procedure of the ASM using an invariant shape representation. The main advantage over exiting outlier handling techniques is that the proposed algorithm is independent of the severity of the outliers, and is robust even when half of the landmarks are incorrectly positioned. Furthermore, the technique uses a geometrically weighted fitness measure for feature point search, which exploits the result of outlier analysis from successive iterations, thus preventing outliers from re-appearing at subsequent iterations. Another contribution of the paper is the introduction of a combined intensity-phase scheme for feature point search, which limits the presence of outliers and reduces the average error of boundary localization significantly. The experimental results on CMR volumetric segmentation of the luminal and outer vessel boundaries of the carotid artery demonstrate the robustness of the algorithm and its potential clinical value. 
Acknowledgements. The authors wish to thank N. Keenan and Prof. D. Pennell from the CMR Unit of Royal Brompton Hospital London for providing the carotid arteries data and the manual delineations.

\section{References}

1. Yang F, Holzapfel G, Schulze-Bauer C, Stollberger R, Thedens D, Bolinger L, Stolpen A, and Sonka M, "Segmentation of wall and plaque in in-vitro vascular MR images," International Journal of Cardiovascular Imaging, vol. 19, pp. 419-428, 2003.

2. Han C, Hatsukami TS, Hwang JN, and Yuan C, "A fast path minimal path active contour model," IEEE Transactions on Medical Imaging, vol. 10, pp. 865-873, 2001.

3. Ladak HM, Thomas JB, Mitchell JR, Rutt BK, and Steinman DA, "A semi-automatic technique for measurement of arterial wall from black blood MRI," Medical Physics, vol. 28, pp. 1098-1107, 2001.

4. Cootes TF, Cooper D, Taylor CJ, and Graham J, "Active shape models - Their training and application," Computer Vision and Image Understanding (CVIU), vol. 61, pp. 38-59, 1995.

5. Rogers M and Graham J, "Robust active shape model search," presented at European Conf. on Computer Vision (ECCV), 2002.

6. Duta N and Sonka M, "Segmentation and interpretation of MR brain images: An improved active shape model," IEEE Transaction on Medical Imaging, vol. 17, pp. 1049-1067, 1998.

7. Behiels G, Vandermeulen D, Maes F, Suetens P, and Dewaele P, "Active shape modelbased segmentation of digital X-ray images," presented at Proc. Medical Image Computing and Computer Assisted Intervention (MICCAI), 1999.

8. Li H and Chutatape O, "Boundary detection of optic disk by a modified ASM method," Pattern Recognition, vol. 36, pp. 2093-2104, 2003.

9. "Outlier detection and handling for robust 3D active shape models search," IEEE Transaction on Medical Imaging, submitted for review.

10. Cootes TF and Taylor CJ, "Active shape model search using local grey-level models: A quantitative evaluation," presented at Proc. British Machine Vision Conf. (BMVC), 1993.

11. Guttman I, Statistical tolerance regions: Classical and Bayesian. London: Griffin, 1970.

12. Crowe LA, Varghese A, Mohiaddin RH, Yang G-Z, and Firmin DN, "Elimination of residual blood flow-related signal in 3D volume-selective TSE arterial wall imaging using velocity-sensitive phase reconstruction," Journal of Magnetic Resonance Imaging, vol. 23, 2006.

13. Ginneken Bv, Frangi AF, Staal JJ, Romeny BMtH, and Viergever MA, "Active shape model segmentation with optimal features," IEEE Transaction on Medical Imaging, vol. 21, pp. 924-933, Aug. 2002.

14. Davatzikos C, Tao X, and Shen D, "Hierarchical active shape models, using the wavelet transform," IEEE Transaction on Medical Imaging, vol. 22, pp. 414-423, Mar. 2003. 